\title{
Covariance characteristics of narrowband periodically non-stationary random signals
}

\author{
Javorskyj I. M. ${ }^{1,3}$, Kurapov P. R. ${ }^{1,2}$, Yuzefovych R. M. ${ }^{1,2}$ \\ ${ }^{1}$ Karpenko Physico-Mechanical Institute of National Academy of Sciences of Ukraine, \\ Laboratory of vibration-based diagnosis, \\ 5 Naukova Str., 79060, Lviv, Ukraine \\ ${ }^{2}$ Lviv Polytechnic National University, Department of Applied Mathematics, \\ 12 S. Bandera Str., 79013, Lviv, Ukraine \\ ${ }^{3}$ UTP University of Sciences and Technology, \\ Institute of Telecommunication and Computer Science, \\ 7 Al. Prof. S. Kaliskiego, 85796, Bydgoszcz, Poland
}

(Received 5 September 2019; Revised 29 October 2019; Accepted 1 November 2019)

\begin{abstract}
Hilbert transform of a narrowband periodically non-stationary random signal (PNRS) is considered. The relations for the covariance components of PNRS and its Hilbert transform are obtained. The dependencies of the covariance properties of Hilbert transform on covariance damping coefficients of modulating processes are analyzed on the basis of the simulated realizations.
\end{abstract}

Keywords: Hilbert transform, narrowband periodically non-stationary random signal, auto-covariance and cross-covariance components, covariance damping coefficients.

2000 MSC: $60 \mathrm{G} 35,94 \mathrm{~A} 12$

UDC: $621.391: 519.72$

DOI: $10.23939 / \mathrm{mmc} 2019.02 .276$

\section{Introduction}

A mathematical model of oscillations in the form of a narrowband process is widely used in various fields of science and engineering: in statistical radiophysics [1-4], in the theory of communication and telemetry [5-9], in technical diagnostics [10-13], in geophysics and oceanology [14, 15], in statistical hydroacoustics [16] etc. For investigation of their properties, the Hilbert transform is usually used

$$
\eta(t)=\mathbb{H}\{\xi(t)\}=\frac{1}{\pi} \int_{-\infty}^{\infty} \frac{\xi(t)}{t-\tau} d \tau,
$$

as it does not change the amplitude of the harmonic components of the input signal $\xi(t)$, but shifts their phases by $-\pi / 2$. The concept of the analytic signal

$$
\zeta(t)=\xi(t)+i \eta(t)=\mu(t) e^{i \psi(t)},
$$

was introduced on the basis of (1) along with the definitions of the envelope $\mu(t)=\left[\xi^{2}(t)+\eta^{2}(t)\right]^{1 / 2}$ and the instantaneous phase $\psi(t)=\arctan \frac{\eta(t)}{\xi(t)}$, and the instantaneous angular frequency

$$
\omega(t)=\frac{d \psi(t)}{d t}=\frac{\xi(t) \eta^{\prime}(t)-\xi^{\prime}(t) \eta(t)}{\mu^{2}(t)} .
$$

The frequency bandwidth $\Delta \omega$ of the signal can be determined using the characteristics of the introduced random processes:

$$
\Delta \omega=\sigma_{\omega}^{2}+\mathbb{E} \dot{\mu}^{2}(t),
$$

here $\mathbb{E}$ is the sign of the mathematical expectation, $\dot{\mu}(t)$ is a derivative of an envelope, $\sigma_{\omega}^{2}(t)=\mathbb{E}[\omega(t)]^{2}-[\mathbb{E} \omega(t)]^{2}$ is the variance of instantaneous frequency. The value $\mathbb{E} \dot{\mu}^{2}(t)$ defines the 
change of the envelope power. The signal $\xi(t)$ is assumed as narrowband if $\Delta \omega^{2} /[\mathbb{E} \omega(t)]^{2} \leqslant 1$, otherwise - broadband. The Hilbert transform is used for both types of signals, but the envelope conception has physical meaning only for narrowband signals $[17,18]$.

Bedrosian's theorem is important in practice [19]: Hilbert transform of the product of low-frequency $\xi_{1}(t)$ and high-frequency $\xi_{2}(t)$ signals equals the product of low-frequency signal and Hilbert transform of high-frequency signal if frequency bands do not overlap:

$$
\mathbb{H}\left\{\xi_{1}(t) \xi_{2}(t)\right\}=\xi_{1}(t) \mathbb{H}\left\{\xi_{2}(t)\right\} .
$$

We choose the mean of the random process $\omega(t)$ as the central frequency of the signal $\mathbb{E} \omega(t)=\omega_{0}$ and set that $\psi(t)=\omega_{0} t-\varphi(t)$. Then the analytical signal (2) can be represented in the form:

$$
\zeta(t)=\left[\xi_{c}(t)-i \xi_{s}(t)\right] e^{i \omega_{0} t},
$$

where

$$
\begin{aligned}
& \xi_{c}(t)=\mu(t) \cos \varphi(t), \\
& \xi_{s}(t)=\mu(t) \sin \varphi(t) .
\end{aligned}
$$

Hence

$$
\begin{aligned}
& \xi(t)=\xi_{c}(t) \cos \omega_{0} t+\xi_{s}(t) \sin \omega_{0} t, \\
& \eta(t)=\xi_{c}(t) \sin \omega_{0} t-\xi_{s}(t) \cos \omega_{0} t .
\end{aligned}
$$

The quantity $\tilde{\mu}(t)=\xi_{c}(t)-i \xi_{s}(t)$ is called a complex envelope, random processes $\xi_{c}(t)$ and $\xi_{s}(t)$ are quadrature components of the signal. They are determined by the expressions:

$$
\begin{aligned}
& \xi_{c}(t)=\xi(t) \cos \omega_{0} t+\eta(t) \sin \omega_{0} t, \\
& \xi_{s}(t)=\xi(t) \sin \omega_{0} t-\eta(t) \cos \omega_{0} t .
\end{aligned}
$$

It follows from E. Bedrosian's theorem, that the random process (4) is Hilbert transform of a signal $\xi(t)$ if the spectra of processes $\xi_{c}(t)$ and $\xi_{s}(t)$ is concentrated in the interval $\left[\omega_{0}-\omega_{m}, \omega_{0}+\omega_{m}\right]$ and $\omega_{m}<\omega_{0}$. In this case the expression for an envelope $\mu(t)$ can be rewritten in the form $\mu(t)=\left[\xi_{c}^{2}(t)+\xi_{s}^{2}(t)\right]^{1 / 2}$. The above-mentioned narrowband signal condition is hard to apply in practice, therefore, it is usually replaced by a condition $\omega_{m} \ll \omega_{0}$ which is obviously much stronger than one in the E. Bedrosian's theorem. In this article the analysis of the dependency of the Hilbert transform properties on the frequency bandwidth of the quadrature components is carried out. However, the main attention is paid to the analysis of those new features and characteristics that it acquires, as well as the overall narrowband signal when we move from a stationary to a periodically non-stationary model.

\section{Narrowband signal as a stationary random process}

Let us suppose that a narrowband signal $\xi(t)$ is described by a stationary random process with a zero mean $m_{\xi}=\mathbb{E} \xi(t)=0$. Then, as it follows from (1), $m_{\eta}=0$ too. Proceeding from the expression (1) and the formula of the inverse Hilbert transform

$$
\xi(t)=-\frac{1}{\pi} \int_{-\infty}^{\infty} \frac{\eta(\tau)}{t-\tau} d \tau
$$

for auto- and cross- covariance functions $R_{\xi}(u)=\mathbb{E} \xi(t) \xi(t+u), R_{\eta}(u)=\mathbb{E} \eta(t) \eta(t+u), R_{\xi \eta}(u)=$ $\mathbb{E} \xi(t) \eta(t+u)$ we obtain:

$$
R_{\xi}(u)=-\frac{1}{\pi} \int_{-\infty}^{\infty} \frac{R_{\xi \eta}(\tau)}{u-\tau} d \tau, \quad R_{\eta \xi}(u)=-\frac{1}{\pi} \int_{-\infty}^{\infty} \frac{R_{\eta}(\tau)}{u-\tau} d \tau
$$

Mathematical Modeling and Computing, Vol. 6, No. 2, pp. 276-288 (2019) 


$$
R_{\xi \eta}(u)=\frac{1}{\pi} \int_{-\infty}^{\infty} \frac{R_{\xi}(\tau)}{u-\tau} d \tau, \quad \quad R_{\eta}(u)=\frac{1}{\pi} \int_{-\infty}^{\infty} \frac{R_{\eta \xi}(\tau)}{u-\tau} d \tau
$$

It follows from these relations that $R_{\xi \eta}(-u)=-R_{\xi \eta}(u)=R_{\eta \xi}(u)$ and $R_{\xi}(u)=R_{\eta}(u)$, i.e. crosscovariance functions of the signal and its Hilbert transform are odd lag functions and have different signs. Their autocovariance functions are equal. Taking into consideration these properties, we find for the auto- and cross-covariance function of the quadrature components:

$$
\begin{aligned}
R_{c}(u)=R_{s}(u) & =R_{\xi}(u) \cos \omega_{0} u+R_{\xi \eta}(u) \sin \omega_{0} u \\
R_{c s}(u) & =R_{\xi}(u) \sin \omega_{0} u-R_{\xi \eta}(u) \cos \omega_{0} u .
\end{aligned}
$$

It follows from formula (6) that $R_{c s}(-u)=-R_{c s}(u)=R_{s c}(u)$.

The covariance function of the analytical signal $R_{\zeta}(u)=\mathbb{E} \bar{\zeta}(t) \zeta(t+u)$ is equal to:

$$
R_{\zeta}(u)=2\left[R_{\xi}(u)+i R_{\xi \eta}(u)\right] .
$$

It is easily seen that cross-covariance function $R_{\xi \eta}(u)$ is the Hilbert transform of autocovariance function $R_{\xi}(u): R_{\xi \eta}(u)=\mathbb{H}\left\{R_{\xi}(u)\right\}$. Taking into account that the transfer function of Hilbert transform $\mathbb{H}(\omega)=-i$ for $\omega>0$ and $\mathbb{H}(\omega)=i$ for $\omega<0$, and also the representation

$$
R_{\xi}(u)=\int_{-\infty}^{\infty} f_{\xi}(\omega) e^{i \omega u} d \omega
$$

where $f_{\xi}(\omega)$ is the power spectral density of signal $\xi(t)$, we obtain:

$$
R_{\xi \eta}(u)=2 \int_{0}^{\infty} f_{\xi}(\omega) \sin \omega u d \omega
$$

And then

$$
R_{\zeta}(u)=4 \int_{0}^{\infty} f_{\xi}(\omega) e^{i \omega u} d \omega .
$$

It follows from the last expression, that

$$
\left|R_{\zeta}(u)\right| \leqslant 2 R_{\xi}(0)
$$

The variance of the analytic signal

$$
R_{\zeta}(0)=4 \int_{0}^{\infty} f_{\xi}(\omega) d \omega
$$

is the mathematical expectation of the square of the envelope signal and it is equal to the sum of the variances of the signal and its Hilbert transform:

$$
R_{\zeta}(0)=\mathbb{E} \mu^{2}(t)=\mathbb{E} \xi^{2}(t)+\mathbb{E} \eta^{2}(t) .
$$

This formula determines also the sum of variances of quadrature components $\xi_{c}(t)$ and $\xi_{s}(t)$ :

$$
R_{\zeta}(0)=R_{c}(0)+R_{s}(0) .
$$

Proceeding from (5) and (6), and also formulae (7) and (8), we can find the dependencies of the auto- and cross-covariance functions and the corresponding power spectral densities of quadrature components on the power spectral density of the signal. Substituting (7) and (8) into (5) we obtain:

$$
R_{c}(u)=R_{s}(u)=2 \int_{0}^{\infty} f_{\xi}(\omega) \cos \left(\omega-\omega_{0}\right) u d \omega=2 \int_{-\omega_{0}}^{\infty} f_{\xi}\left(\omega+\omega_{0}\right) \cos \omega u d \omega .
$$


The spectral density of narrowband process is concentrated in narrow intervals $\left[\omega_{0}-\Delta \omega, \omega_{0}+\Delta \omega\right]$ and $\left[-\omega_{0}-\Delta \omega,-\omega_{0}+\Delta \omega\right]$, therefore, the last integral can be rewritten in the form:

$$
R_{c}(u) \cong 2 \int_{-\omega_{0}}^{\infty} \tilde{f}_{\xi}\left(\omega+\omega_{0}\right) d \omega,
$$

where $\tilde{f}_{\xi}\left(\omega+\omega_{0}\right)$ is the component of the spectrum in the positive frequency domain shifted by the amount $-\omega_{0}$. Then $f_{c}(\omega)=f_{s}(\omega) \cong 2 \tilde{f}_{\xi}\left(\omega+\omega_{0}\right)$, i.e. the power spectral densities of the quadrature components are equal to the component of the spectrum of the signal in the positive frequencies domain multiplied by 2 , shifted to the left by carrier frequency $\omega_{0}$.

After substituting (7) and (8) into (6) we obtain:

$$
\begin{aligned}
R_{c s}(u) & =2 \int_{0}^{\infty} f_{\xi}(\omega) \sin \left(\omega-\omega_{0}\right) u d \omega=2 \int_{-\omega_{0}}^{\infty} f_{\xi}\left(\omega+\omega_{0}\right) \sin (\omega) u d \omega \\
& \cong 2 \int_{-\omega_{0}}^{\infty} \tilde{f}_{\xi}\left(\omega+\omega_{0}\right) \sin (\omega) u d \omega .
\end{aligned}
$$

It follows from this expression that the cross-covariance function of quadrature components is not equal to zero only in the case when $\tilde{f}_{\xi}\left(\omega+\omega_{0}\right)$ contains an odd component. It is easily seen from (9) that $R_{c s}(u)$ is odd lag function and $R_{c s}(0)=0$ if $u=0$. Thus, the quadrature components of the stationary narrowband signal are uncorrelated at the same moments of time.

\section{The Hilbert transform of periodically non-stationary narrowband signal}

As it was noted above, the auto-covariance functions of the quadrature components of the narrowband stationary random process are equal, and their cross-covariance function is an odd lag function. Assuming now that these conditions are not satisfied and we will analyze the covariance and spectral properties of the Hilbert transform of such a narrowband signal.

If the mean of quadrature components $m_{c}=\mathbb{E} \xi_{c}(t)$ and $m_{s}=\mathbb{E} \xi_{s}(t)$ are not equal to zero, then

$$
m_{\xi}(t)=m_{c} \cos \omega_{0} t+m_{s} \sin \omega_{0} t .
$$

For the mean function of the Hilbert transform we obtain:

$$
m_{\eta}(t)=\frac{1}{\pi} \int_{-\infty}^{\infty} \frac{m_{\xi}(t)}{t-\tau} d \tau=m_{c} \sin \omega_{0} t-m_{s} \cos \omega_{0} t
$$

The mean function of the analytic signal (2) has the form:

$$
m_{\zeta}(t)=m_{\xi}(t)+i m_{\eta}(t)=m e^{i \omega t}
$$

where $m=m_{c}-i m_{s}$.

The covariance function $b_{\xi}(t, u)=\mathbb{E}\left[\xi(t)-m_{\xi}(t)\right]\left[\xi(t+u)-m_{\xi}(t+u)\right]$ of periodically nonstationary narrowband signal (3) is determined by formula [12]:

$$
b_{\xi}(t, u)=B_{0}^{(\xi)}(u)+C_{2}^{(\xi)}(u) \cos 2 \omega_{0} t+S_{2}^{(\xi)} \sin 2 \omega_{0} t=B_{0}^{(\xi)}(u)+\sum_{k= \pm 2} B_{k}^{(\xi)}(u) e^{i k \omega_{0} t}
$$

where

$$
\begin{aligned}
& B_{0}^{(\xi)}(u)=\frac{1}{2}\left[R_{c}(u)+R_{s}(u)\right] \cos \omega_{0} u+R_{c s}^{-}(u) \sin \omega_{0} u \\
& B_{2}^{(\xi)}(u)=\frac{1}{2}\left[\frac{1}{2}\left[R_{c}(u)-R_{s}(u)\right]-i R_{c s}^{+}(u)\right] e^{i \omega_{0} u} .
\end{aligned}
$$

Mathematical Modeling and Computing, Vol. 6, No. 2, pp. 276-288 (2019) 
Here $B_{2}^{(\xi)}(u)=\frac{1}{2}\left[C_{2}^{(\xi)}(u)-i S_{2}^{(\xi)}(u)\right]$, and also $R_{c s}^{+}(u)$ and $R_{c s}^{-}(u)$ are even and odd parts of the crosscovariance function $R_{c s}=R_{c s}^{+}(u)+R_{c s}^{-}(u)$. Obviously, that $B_{-2}^{(\xi)}(u)=\overline{\mathrm{B}}_{2}^{(\xi)}(u)$, where "-" is a sign of conjugation. Thus, the random signal (3) is PNRS [11,12,14,15]. The quantities $B_{k}^{(\xi)}(u)$ are called covariance components $[12,15]$. The zero covariance component $B_{0}^{(\xi)}(u)$, which is a time-averaged value of the covariance function, has all the properties of the covariance function of the stationary random process. It is called the covariance function of the stationary approximation of a periodically non-stationary random process [12].

Since $b_{\xi}(t,-u)=b_{\xi}(t-u, u)$, then $B_{k}(-u)=B_{k}(u) e^{-i k \omega_{0} u}$. Thus, the zero covariance component is the even lag function: $B_{0}(-u)=B_{0}(u)$. For the second cosine and sine covariance components from the equality $B_{2}(-u)=B_{2}(u) e^{-i 2 \omega_{0} u}$ we obtain:

$$
\begin{aligned}
C_{2}^{(\xi)}(-u) & =C_{2}^{(\xi)}(u) \cos 2 \omega_{0} u-S_{2}^{(\xi)}(u) \sin 2 \omega_{0} u, \\
S_{2}^{(\xi)}(-u) & =C_{2}^{(\xi)}(u) \sin 2 \omega_{0} u+S_{2}^{(\xi)}(u) \cos 2 \omega_{0} u .
\end{aligned}
$$

The instantaneous spectral density

$$
f_{\xi}(\omega, t)=\frac{1}{2 \pi} \int_{-\infty}^{\infty} b_{\xi}(t, u) e^{-i \omega u} d u
$$

of the signal (3) is equal to:

$$
f_{\xi}(\omega, t)=\sum_{k=0, \pm 2} f_{k}^{(\xi)}(\omega) e^{i k \omega_{0} t}
$$

where

$$
f_{k}^{(\xi)}(\omega)=\frac{1}{2 \pi} \int_{-\infty}^{\infty} B_{k}^{(\xi)}(u) e^{-i \omega u} d u
$$

The quantities (15) are called the spectral components of PNRS (also cyclic covariance function or cyclic spectral densities [6]). The zero spectral components $f_{0}(\omega)$ is even frequency function: $f_{0}(-\omega)=f_{0}(\omega)$. It determines the time averaged power of PNRS. The second spectral components satisfy the equalities:

$$
f_{2}(-\omega)=f_{2}\left(\omega+2 \omega_{0}\right)=\bar{f}_{-2}(\omega) .
$$

Proceeding from (11) and (12) we obtain:

$$
\begin{gathered}
f_{0}(\omega)=\frac{1}{4}\left[f_{c}\left(\omega+\omega_{0}\right)+f_{s}\left(\omega+\omega_{0}\right)+f_{c}\left(\omega-\omega_{0}\right)+f_{s}\left(\omega-\omega_{0}\right)\right]+\frac{1}{2}\left[f_{c s}^{-}\left(\omega+\omega_{0}\right)-f_{c s}^{-}\left(\omega-\omega_{0}\right)\right], \\
f_{2}(\omega)=\frac{1}{4}\left[f_{c}\left(\omega-\omega_{0}\right)-f_{s}\left(\omega-\omega_{0}\right)\right]-\frac{i}{2}\left[f_{c s}^{+}\left(\omega-\omega_{0}\right)\right]
\end{gathered}
$$

where

$$
\begin{gathered}
f_{c, s}(\omega)=\frac{1}{\pi} \int_{0}^{\infty} R_{c, s}(u) \cos \omega u d u \\
f_{c, s}^{-}(\omega)=\frac{1}{\pi} \int_{0}^{\infty} R_{c, s}^{-}(u) \sin \omega u d u, \quad f_{c, s}^{+}(\omega)=\frac{1}{\pi} \int_{0}^{\infty} R_{c, s}^{+}(u) \cos \omega u d u
\end{gathered}
$$

Since

$$
\left|f_{c s}(\omega)\right|^{2} \leqslant f_{c}(\omega) f_{s}(\omega)
$$

then the spectrum bandwidth of PNRS is determined by the zero spectral components. Below we shall call PNRS narrowband if

$$
f_{0}(\omega)= \begin{cases}f_{0}(\omega), & \omega \in\left[\omega_{0}-\Delta \omega, \omega_{0}+\Delta \omega\right] \\ 0, & \omega \notin\left[\omega_{0}-\Delta \omega, \omega_{0}+\Delta \omega\right]\end{cases}
$$

Mathematical Modeling and Computing, Vol. 6, No. 2, pp. 276-288 (2019) 
where $\Delta \omega<\omega_{0}$. It is mean that the value of $f_{c, s}(\omega)$ are concentrated in the interval $\left[-\omega_{0}, \omega_{0}\right]$ and for narrowband PNRS condition of the Bedrosian's theorem is satisfied. Note that the value of the second spectral component of narrowband PNRS as follows from (17) are concentrated in the interval $\left(0,2 \omega_{0}\right)$.

The auto-covariance and cross-covariance functions of the signal and its Hilbert transform are connected by relations [20]:

$$
\begin{aligned}
b_{\xi \eta}(t, u) & =\frac{1}{\pi} \int_{-\infty}^{\infty} \frac{b_{\eta}(t+u, \tau)}{\tau+u} d \tau, \\
b_{\xi}(t, u) & =\frac{1}{\pi} \int_{-\infty}^{\infty} \frac{b_{\xi \eta}(t+u, \tau)}{\tau+u} d \tau, \\
b_{\eta \xi}(t, u) & =-\frac{1}{\pi} \int_{-\infty}^{\infty} \frac{b_{\xi}(t+u, \tau)}{\tau+u} d \tau, \\
b_{\eta}(t, u) & =-\frac{1}{\pi} \int_{-\infty}^{\infty} \frac{b_{\eta \xi}(t+u, \tau)}{\tau+u} d \tau .
\end{aligned}
$$

Proposition 1. A periodically non-stationary random signal, the covariance function of which is determined by the relations (10)-(12), and its Hilbert transform are jointly periodically non-stationary random processes and their auto- and cross- covariance component are connected by expressions:

$$
\begin{aligned}
B_{k}^{(\eta)}(u) & =\int_{-\infty}^{\infty} h(u-\tau) B_{k}^{(\eta \xi)}(\tau) d \tau, \\
B_{k}^{(\eta \xi)}(u) & =-\int_{-\infty}^{\infty} h(u-\tau) B_{k}^{(\eta)}(\tau) d \tau, \\
B_{k}^{(\xi)}(u) & =-\int_{-\infty}^{\infty} h(u-\tau) B_{k}^{(\xi \eta)}(\tau) d \tau, \\
B_{k}^{(\xi \eta)}(u) & =\int_{-\infty}^{\infty} h(u-\tau) B_{k}^{(\xi)}(\tau) d \tau,
\end{aligned}
$$

where $h(\tau)=(\pi \tau)^{-1}$ is the pulse response of the Hilbert transform, i.e. the covariance components $B_{k}^{(\eta)}(u)$ and $B_{k}^{(\eta \xi)}(u)$, and also $B_{k}^{(\xi \eta)}(u)$ and $B_{k}^{(\xi)}(u)$ are Hilbert transform pairs.

Proof. After substituting into formula (20) the representation (10) we obtain

$$
b_{\eta \xi}(t, u)=\sum_{k=0, \pm 2} e^{i k \omega_{0} t}\left[-\frac{1}{\pi} \int_{-\infty}^{\infty} \frac{B_{k}^{(\xi)}(\tau)}{\tau+u} d \tau\right] e^{i k \omega_{0} u} .
$$

It follows from this equality that the cross-covariance function $b_{\eta \xi}(t, u)$ varies with time periodically, and its Fourier coefficients are determined by the formula

$$
B_{k}^{(\eta \xi)}(u)=-\frac{e^{i k \omega_{0} u}}{\pi} \int_{-\infty}^{\infty} \frac{B_{k}^{(\xi)}(\tau)}{\tau+u} d \tau,
$$

hence:

$$
B_{k}^{(\eta \xi)}(-u)=e^{-i k \omega_{0} u} \int_{-\infty}^{\infty} h(u-\tau) B_{k}^{(\xi)}(\tau) d \tau
$$

It's easily seen that $b_{\xi \eta}(t,-u)=b_{\eta \xi}(t-u, u)$. Then $B_{k}^{(\xi \eta)}(-u)=B_{k}^{(\eta \xi)}(u) e^{-i k \omega_{0} u}$. Taking into account the last equality, we obtain the formula (25).

Let us substitute the cross-covariance function

$$
b_{\xi \eta}(t, u)=\sum_{k=0, \pm 2} B_{k}^{(\xi \eta)}(u) e^{i k \omega_{0} t}
$$


into formula (19). Then

or

$$
B_{k}^{(\xi)}(u)=\frac{e^{i k \omega_{0} u}}{\pi} \int_{-\infty}^{\infty} \frac{B_{k}^{(\xi \eta)}(\tau)}{\tau+u} d \tau
$$

$$
B_{k}^{(\xi)}(-u) e^{i k \omega_{0} u}=-\int_{-\infty}^{\infty} h(u-\tau) B_{k}^{(\xi \eta)}(\tau) d \tau
$$

Taking into account the equality $B_{k}^{(\xi)}(-u) e^{i k \omega_{0} u}=B_{k}^{(\xi)}(u)$, we obtain formula (24).

It follows from expression (21) that

$$
B_{k}^{(\eta)}(u)=-\frac{e^{i k \omega_{0} u}}{\pi} \int_{-\infty}^{\infty} \frac{B_{k}^{(\eta \xi)}(\tau)}{\tau+u} d \tau
$$

using that $B_{k}^{(\eta)}(-u) e^{i k \omega_{0} u}=B_{k}^{(\eta)}(u)$ we come to (22). Taking into consideration the relation (18), the representation

$$
b_{\eta}(t, u)=\sum_{k=0, \pm 2} B_{k}^{(\eta)}(u) e^{i k \omega_{0} t}
$$

and equality $B_{k}^{(\xi \eta)}(u)=B_{k}^{(\eta \xi)}(-u) e^{i k \omega_{0} u}$ we arrive the formula (23). To simplify the further analysis, we rewrite the relations $(22)-(25)$ in the frequency domain. Fourier transforms of cross-covariance components

$$
f_{k}^{(\xi \eta)}(\omega)=\frac{1}{2 \pi} \int_{-\infty}^{\infty} B_{k}^{(\xi \eta)}(u) e^{-i k \omega u} d \omega
$$

are called cross-spectral components $[12,15]$. They have the following properties:

$$
f_{k}^{(\xi \eta)}(-\omega)=f_{k}^{(\eta \xi)}\left(\omega+k \omega_{0}\right)=\bar{f}_{-k}^{(\xi \eta)}(\omega) .
$$

Using (15) and (22)-(26), we obtain:

$$
\begin{aligned}
f_{k}^{(\eta)}(\omega) & =\mathbb{H}(\omega) f_{k}^{(\eta \xi)}(\omega), \\
f_{k}^{(\eta \xi)}(\omega) & =-\mathbb{H}(\omega) f_{k}^{(\eta)}(\omega), \\
f_{k}^{(\xi)}(\omega) & =-\mathbb{H}(\omega) f_{k}^{(\xi \eta)}(\omega), \\
f_{k}^{(\xi \eta)}(\omega) & =\mathbb{H}(\omega) f_{k}^{(\xi)}(\omega) .
\end{aligned}
$$

Proposition 2. The zero covariance components of the periodically nonstationary narrowband signal (3) and its Hilbert transform are equal and their zero cross-covariance components differ only by a sign. They are odd lag functions and are determined by the zero spectral component $f_{0}^{(\xi)}(\omega)$ of the signal:

$$
B_{0}^{(\xi \eta)}(u)=2 \int_{0}^{\infty} f_{0}^{(\xi)}(\omega) \sin \omega u d \omega
$$

Proof. It follows from equation (27) that $f_{0}^{(\eta \xi)}(\omega)=\bar{f}_{0}^{(\xi \eta)}(\omega)$. Using (30) we have $f_{0}^{(\eta \xi)}(\omega)=$ $-\mathbb{H}(\omega) f_{0}^{(\xi)}(\omega)$. Substitution the last equality into (28), we come to expression $f_{0}^{(\eta)}(\omega)=$ $-\mathbb{H}(\omega) \mathbb{H}(\omega) f_{0}^{(\xi)}(\omega)=f_{0}^{(\xi)}(\omega)$ and $B_{0}^{(\eta)}(u)=B_{0}^{(\xi)}(u)$. Proceeding from (30) for a zero cross-covariance component $B_{0}^{(\xi \eta)}(u)$ we obtain:

$$
\begin{aligned}
B_{0}^{(\xi \eta)}(u) & =\int_{-\infty}^{\infty} \mathbb{H}(\omega) f_{0}^{(\xi)}(\omega) e^{i \omega u} d \omega=i \int_{-\infty}^{\infty} \operatorname{sign}(\omega) f_{0}^{(\xi)}(\omega) e^{i \omega u} d \omega \\
& =\int_{-\infty}^{\infty} f_{0}^{(\xi)}(\omega)\left(-i e^{i \omega u}+i e^{-i \omega u}\right) d \omega=2 \int_{-\infty}^{\infty} f_{0}^{(\xi)}(\omega) \sin \omega u d \omega
\end{aligned}
$$


Since $f_{0}^{(\xi)}(\omega)=f_{0}^{(\eta)}(\omega)$ and expressions (29) and (30) differ only by the sign, therefore $B_{0}^{(\xi \eta)}(u)=$ $-B_{0}^{(\eta \xi)}(u)$. It follows from (31) that the cross-covariance components are the odd lag function.

Let us now consider the properties of the second auto- and cross- covariance components.

Proposition 3. The second covariance components of the periodically non-stationary narrowband signal and its Hilbert transform have the different $\operatorname{sign} B_{2}^{(\eta)}(u)=-B_{2}^{(\xi)}(u)$ and their second crosscovariance components are symmetric and related with the second covariance component of the signal by the relation:

$$
B_{2}^{(\xi \eta)}(u)=B_{2}^{(\eta \xi)}(u)=-i B_{2}^{(\xi)}(u) .
$$

Proof. Taking into consideration the relations $\mathbb{H}(-\omega)=\mathbb{H}(\omega), f_{2}^{(\eta \xi)}(-\omega)=f_{2}^{(\xi \eta)}\left(\omega+2 \omega_{0}\right)$ and (28) we obtain:

$$
\begin{aligned}
B_{2}^{(\eta)}(u) & =\int_{-\infty}^{\infty} \mathbb{H}(\omega) f_{2}^{(\eta \xi)}(\omega) e^{i \omega u} d \omega=-\int_{-\infty}^{\infty} \mathbb{H}(\omega) f_{2}^{(\eta \xi)}(-\omega) e^{-i \omega u} d \omega \\
& =-\int_{-\infty}^{\infty} \mathbb{H}(\omega) f_{2}^{(\xi \eta)}\left(\omega+2 \omega_{0}\right) e^{-i \omega u} d \omega
\end{aligned}
$$

Now introduce the variable $\nu=\omega+2 \omega_{0}$. Using the equality (30), we have:

$$
B_{2}^{(\eta)}(u)=-e^{i 2 \omega_{0} u} \int_{-\infty}^{\infty} f_{2}^{(\xi)}(\omega) \mathbb{H}\left(\omega-2 \omega_{0}\right) \mathbb{H}(\omega) e^{-i \omega u} d \omega
$$

Since $B_{2}^{(\eta)}(-u)=B_{2}^{(\eta)}(u) e^{-i 2 \omega_{0} u}$, then

$$
B_{2}^{(\eta)}(u)=-\int_{-\infty}^{\infty} f_{2}^{(\xi)}(\omega) \mathbb{H}\left(\omega-2 \omega_{0}\right) \mathbb{H}(\omega) e^{i \omega u} d \omega
$$

and hence

$$
f_{2}^{(\eta)}(\omega)=-\mathbb{H}\left(\omega-2 \omega_{0}\right) \mathbb{H}(\omega) f_{2}^{(\xi)}(\omega)
$$

We substitute the last equality into the formula (29). Then

$$
f_{2}^{(\eta \xi)}(\omega)=\mathbb{H}(\omega) \mathbb{H}(\omega) \mathbb{H}\left(\omega-2 \omega_{0}\right) f_{2}^{(\xi)}(\omega)=-\mathbb{H}\left(\omega-2 \omega_{0}\right) f_{2}^{(\xi)}(\omega)
$$

Taking into account that

$$
-\mathbb{H}(\omega) \mathbb{H}\left(\omega-2 \omega_{0}\right)=\left\{\begin{aligned}
1, & \omega \in(-\infty, 0] \\
-1, & \omega \in\left(0,2 \omega_{0}\right], \\
1, & \omega \in\left(2 \omega_{0}, \infty\right)
\end{aligned}\right.
$$

we rewrite the integral (32) in the form

$$
\begin{aligned}
B_{2}^{(\eta)}(u) & =\int_{-\infty}^{0} f_{2}^{(\xi)}(\omega) e^{i \omega u} d \omega-\int_{0}^{2 \omega_{0}} f_{2}^{(\xi)}(\omega) e^{i \omega u} d \omega+\int_{2 \omega_{0}}^{\infty} f_{2}^{(\xi)}(\omega) e^{i \omega u} d \omega \\
& =\int_{-\infty}^{\infty} f_{2}^{(\xi)}(\omega) e^{i \omega u} d \omega-2 \int_{0}^{2 \omega_{0}} f_{2}^{(\xi)}(\omega) e^{i \omega u} d \omega .
\end{aligned}
$$

The second spectral component is determined by the formula (17). The spectral densities $f_{c}\left(\omega-\omega_{0}\right)$, $f_{s}\left(\omega-\omega_{0}\right)$ and $f_{c s}^{+}\left(\omega-\omega_{0}\right)$ for narrowband PNRS are concentrated in $\left(\omega_{0}-\Delta \omega, \omega_{0}+\Delta \omega\right)$, so we can put

$$
B_{2}^{(\eta)}(u) \cong-\int_{0}^{2 \omega_{0}} f_{2}^{(\xi)}(\omega) e^{i \omega u} d \omega=-\int_{-\infty}^{\infty} f_{2}^{(\xi)}(\omega) e^{i \omega u} d \omega=-B_{2}^{(\xi)}(u) .
$$


Using the relation (16), (30) and (33) for the cross-covariance components we have that:

$$
\begin{aligned}
& B_{2}^{(\xi \eta)}(u)=i \int_{0}^{\infty}\left[f_{2}^{(\xi)}\left(\omega+2 \omega_{0}\right) e^{-i \omega u}-f_{2}^{(\xi)}(\omega) e^{i \omega u}\right] d \omega \\
& B_{2}^{(\eta \xi)}(u)=-i\left[2 \int_{0}^{2 \omega_{0}} f_{2}^{(\xi)}(\omega) e^{i \omega u} d \omega+\int_{0}^{\infty}\left[f_{2}^{(\xi)}\left(\omega+2 \omega_{0}\right)-f_{2}^{(\xi)}(\omega)\right] e^{i \omega u} d \omega\right] .
\end{aligned}
$$

Hence for the narrowband process:

$$
\begin{aligned}
& B_{2}^{(\xi \eta)}(u)=-i \int_{0}^{2 \omega_{0}} f_{2}^{(\xi)}(\omega) e^{i \omega u} d \omega \cong-i \int_{-\infty}^{\infty} f_{2}^{(\xi)}(\omega) e^{i \omega u} d \omega \\
& B_{2}^{(\eta \xi)}(u)=-i \int_{0}^{2 \omega_{0}} f_{2}^{(\xi)}(\omega) e^{i \omega u} d \omega \cong-i \int_{-\infty}^{\infty} f_{2}^{(\xi)}(\omega) e^{i \omega u} d \omega .
\end{aligned}
$$

So $B_{2}^{(\xi \eta)}(u)=B_{2}^{(\eta \xi)}(u)=-i B_{2}^{(\xi)}(u)$.

\section{Numerical results}

To analyze the dependency of covariance properties for Hilbert transform on the rate of covariance damping coefficients $\alpha_{i}$ of the signal we use the processing results of the simulated random sequences (see Fig. 1):

$$
\xi(n h)=\xi^{c}(n h) \cos \left(\frac{2 \pi}{T} n h\right)+\xi^{s}(n h) \sin \left(\frac{2 \pi}{T} n h\right) .
$$

We have chosen the following approximations:

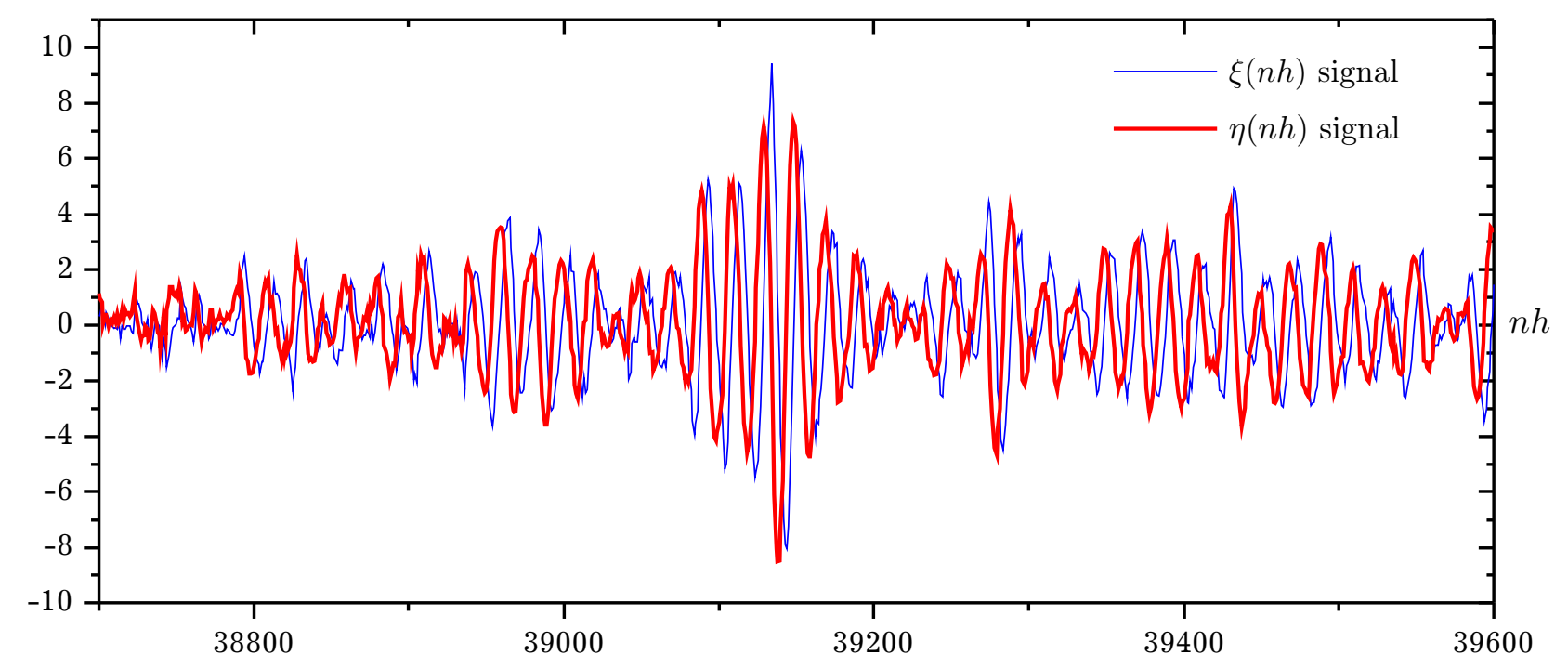

Fig. 1. The simulated realization $\xi(n h)$ and its Hilbert transform $\eta(n h)$ for $\alpha_{1}=0.02$.

$$
B_{c}(u)=D_{c} e^{-\alpha_{c}|u|}, \quad B_{s}(u)=D_{s} e^{-\alpha_{s}|u|}, \quad B_{c s}(\tau)=D_{c s} e^{-\alpha_{c s}|u|},
$$

and also the folloving values of parameters:

$$
T=20, \quad D_{c}=4, \quad D_{s}=1, \quad D_{c s}=1, \quad m_{c}=m_{s}=m_{c s}=0 .
$$


The realization was simulated for the coefficients $\alpha_{c}=\alpha_{s}=2 \alpha_{c s}=\alpha_{i}$, herewith we consider cases when $\alpha_{1}=0.02, \alpha_{2}=0.04$, $\alpha_{3}=0.08, \alpha_{4}=0.1$.

If we chose the bandwidth of the signal on the basis of equality $f_{0}\left(\omega_{m}\right)=0.05 f_{0}\left(\omega_{0}\right)$, where $\omega_{m}$ is the cut-off frequency, then we have $\omega_{m}=0.28 \omega_{0}$ and $\omega_{m}=0.59 \omega_{0}$ for the first and second coefficient values and $\omega_{m}=1.1 \omega_{0}$, $\omega_{m}=1.26 \omega_{0}$ for next.

Thus, in the last cases the conditions of the Bedrosian's theorem are not satisfied and then we can expect of the estimators for Hilbert transform of covariance component

$$
\begin{array}{r}
\hat{B}_{0}^{(\eta)}(r h)=\frac{1}{K} \sum_{n=0}^{K-1} \stackrel{\circ}{\eta}(n h) \stackrel{\circ}{\eta}[(n+r) h], \\
\left\{\begin{array}{l}
\hat{C}_{2}^{(\eta)}(r h) \\
\hat{S}_{2}^{(\eta)}(r h)
\end{array}\right\}=\frac{1}{K} \sum_{n=0}^{K-1} \stackrel{\circ}{\eta}(n h) \stackrel{\circ}{\eta}[(n+r) h]\left\{\begin{array}{l}
\cos \frac{4 \pi}{T} n h \\
\sin \frac{4 \pi}{T} n h
\end{array}\right\}
\end{array}
$$

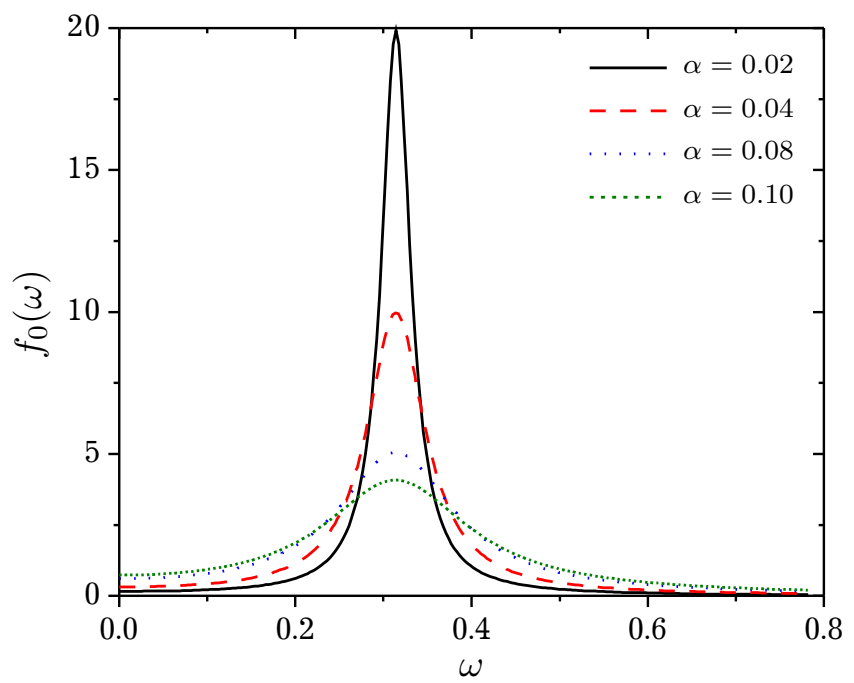

Fig. 2. The zero $^{\text {th }}$ spectral component of the signal: 1) $\left.\left.\left.\alpha_{1}=0.02,2\right) \alpha_{2}=0.04,3\right) \alpha_{3}=0.08,4\right) \alpha_{4}=0.10$.

differ from the theoretical forms. It was confirmed by the calculations, which was carried out. For the first values $\alpha_{i}$ difference between the covariance component estimators for the signal and its Hilbert transform is not significant (see Tables 1,2, Figs. 3, 4).

Table 1. The relative errors $\delta\left[\hat{B}_{0}(r h)\right]$ for different coefficient $\alpha_{i}$.

\begin{tabular}{|c|c|c|c|c|}
\hline & $\alpha_{1}=0.02$ & $\alpha_{2}=0.04$ & $\alpha_{3}=0.08$ & $\alpha_{4}=0.1$ \\
\hline$\delta\left[\hat{B}_{0}(r h)\right]$ & $1.40877 \mathrm{E}-05$ & $6.97 \mathrm{E}-06$ & $3.22 \mathrm{E}-05$ & 0.00010754 \\
\hline$\delta\left[\hat{B}_{2}^{c}(r h)\right]$ & 0.000482 & 0.001509 & 0.005104 & 0.037413 \\
\hline$\delta\left[\hat{B}_{2}^{s}(r h)\right]$ & 0.000134 & 0.000269 & 0.0048406 & 0.095018 \\
\hline
\end{tabular}

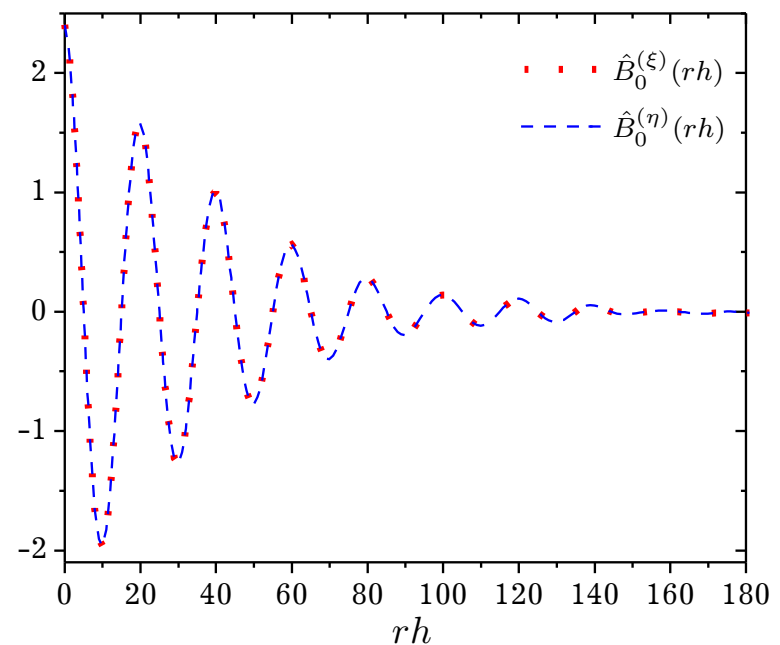

Fig. 3. The estimators of the zero ${ }^{\text {th }}$ covariance component for the signal and its Hilbert transform.

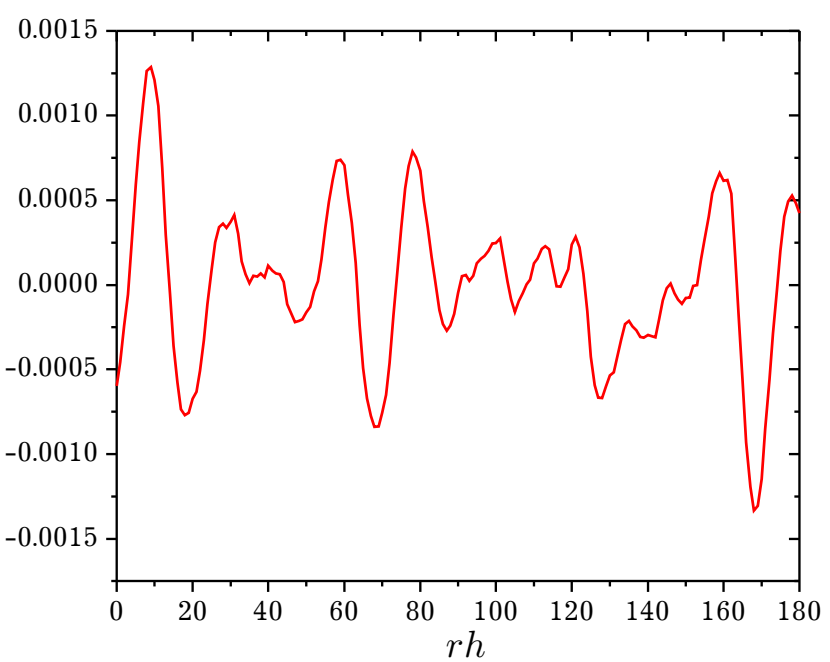

Fig. 4. The deference $\hat{B}^{\xi}{ }_{0}(\tau h)-\hat{B}^{\eta}{ }_{0}(\tau h)$ in the dependency on lag. 
Table 2. The estimators of the second covariance component.

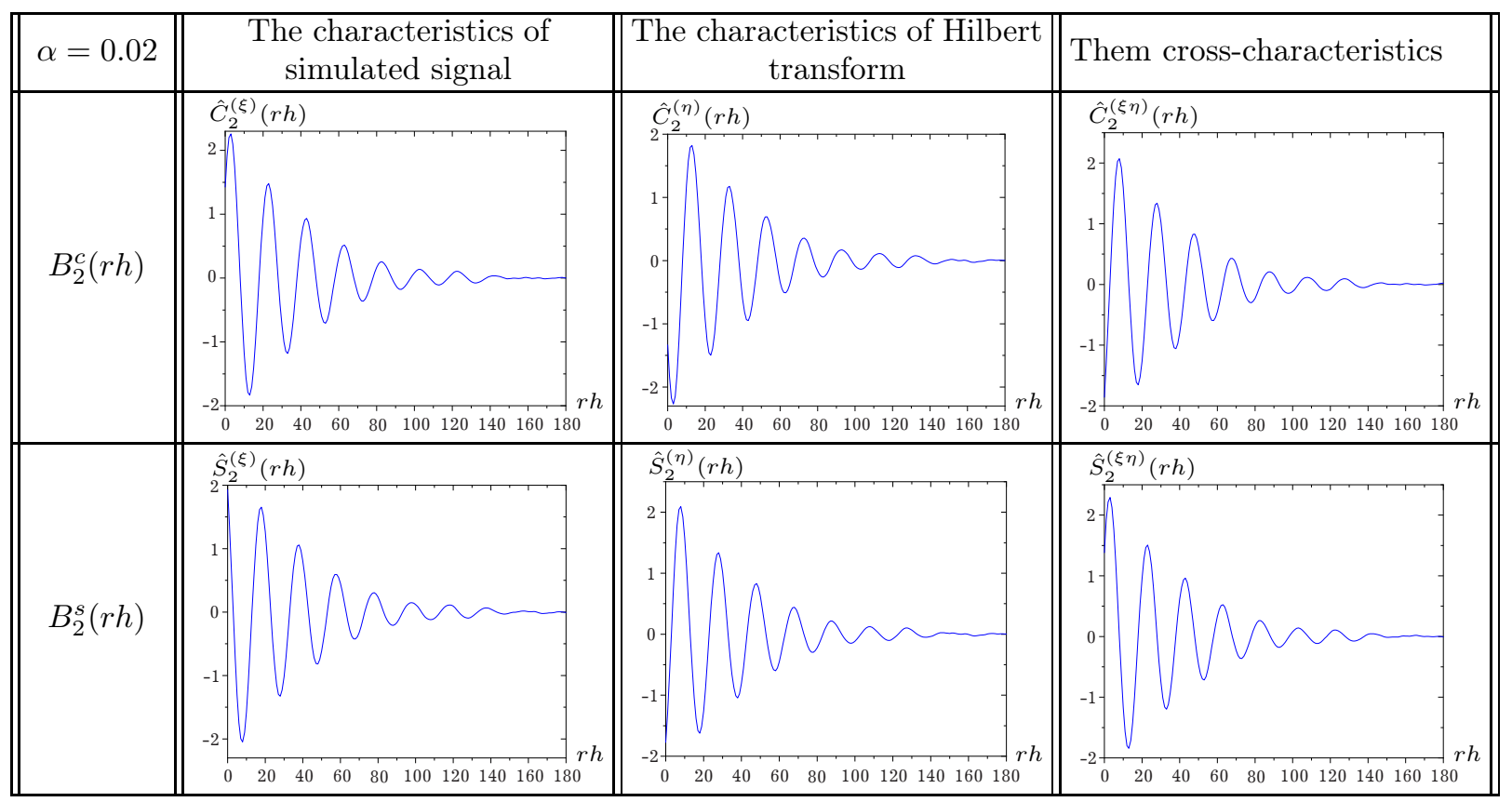

As we see from Table 1 the relative error of the zero ${ }^{\text {th }}$ component increases slowly as $\alpha_{i}$ increas.

$$
\delta\left[\hat{B}_{0}^{(\eta)}\left(r_{\max } h\right)\right]=\frac{1}{\left(r_{\max }+1\right) h \hat{B}_{0}^{(\eta)}(0)} \sum_{r=0}^{r_{\max }}\left[\hat{B}_{0}^{(\xi)}\left(r_{\max } h\right)-\hat{B}_{0}^{(\eta)}\left(r_{\max } h\right)\right]^{2} .
$$

However the relative error of the second component estimators calculated similarly yet achieves the value 0.07 . The experimental results allow to conclude that the satisfaction of the inequality $\alpha<3 \omega_{0}$ provides the relative errors that are not larger than 0.04 .

\section{Conclusions}

The covariance properties of Hilbert transform of a narrowband PNRS are analyzed in this article. It is shown that PNRS and its Hilbert transform are jointly periodically non-stationary random processes and their auto- and cross- covariance components are Hilbert transform pairs. The zero auto-covariance of the signal $\xi(t)$ and its Hilbert transform $\eta(t)$ are equal and the second components differ only by sign: $B_{0}^{(\eta)}(u)=B_{0}^{(\xi)}(u), B_{2}^{(\eta)}(u)=-B_{2}^{(\xi)}(u)$. The zero cross-covariance component have the different sign $B_{0}^{(\xi \eta)}(u)=-B_{0}^{(\eta \xi)}(u)$, they are odd lag functions $B_{0}^{(\xi \eta)}(-u)=-B_{0}^{(\xi \eta)}(u)$ and are determinate by onesided sine transform of the zero spectral component of the signal: $B_{0}^{(\xi \eta)}(u)=2 \int_{0}^{\infty} f_{0}^{(\xi)}(\omega) \sin \omega u d \omega$. The second cross-covariance components are symmetric $B_{2}^{(\xi \eta)}(u)=-B_{2}^{(\eta \xi)}(u)$. And they are related to the second covariance component of the signal by the equality $B_{2}^{(\xi \eta)}(u)=-i B_{2}^{(\xi)}(u)$. On the basis of statistical processing of the simulated realization of the PNRS quadrature model the influence of the damping covariance coefficients of the modulating processes on the covariance properties of Hilbert transform was analyzed. The numerical values of the mean square errors are obtained for the covariance component for increasing sequence of covariance damping coefficients $\alpha$. The empirical inequality for damping coefficient is obtained.

[1] Rytov S. M., Kravtsov Yu. A., Tatarskii V. I. Principles of Statistical Radiophysics 1 - Elements of Random Process Theory. Berlin, Heidelberg, Springer-Verlag (1987). 
[2] Deutsch R. Nonlinear transformations of nonlinear Processes. New York, Prentice Hall, Englewood Cliffs (1962).

[3] Tihonov V. I. Nelinejnye preobrazovanija sluchajnyh processov. Moskva, Radio i Sviaz (1986), (in Russian).

[4] Levin B. R. Teoreticheskie osnovy statisticheskoj radiotehniki. Moskva, Sovetskoe radio (1974), (in Russian).

[5] Papoulis A. Random modulation: A review. IEEE Transactions on Acoustics, Speech, and Signal Processing. 31 (1), 96-105 (1983).

[6] Garder W. A. Introduction to random processes with application to signal and systems. New York, Macmillan Pub. Co (1985).

[7] Bendat J. S., Piersol A. G. Random data: analysis and measurement procedures. Wiley (2010).

[8] Gabor D. Theory of communication. Journal of the Institution of Electrical Engineers - Part III: Radio and Communication Engineering. 93 (26), 429-441 (1946).

[9] Oppenheim A. V., Schafer R. W. Digital signal processing. New York, Prentice Hall (1975).

[10] Randall R. B., Antoni I., Chobsaard S. The relation between spectral correlation and envelope analysis. Mechanical Systems and Signal Processing. 15 (5), 945-962 (2001).

[11] Javorskyi I., Kravets I., Matsko I., Yuzefovych R. Periodically correlated random processes: Application in early diagnostics of mechanical systems. Mechanical Systems and Signal Processing. 83, 406-438 (2017).

[12] Javorskyi I. Mathematical models and analisys of stochastic oscillations. Lviv, Karpenko PhysicoMechanical Institute (2013), (in Ukraine).

[13] Feldman M. Hilbert transform applications in mechanical vibration. John Wiley (2011).

[14] Rozhkow V. Metody verojatnostnogo analiza okeanologicheskih processov. Leningrad, Gidrometeoizdat (1974), (in Russian).

[15] Dragan Y., Rozhkow V., Javorskyj I. Metody verojatnostnogo analiza ritmiki okeanologicheskih processov. Leningrad, Gidrometeoizdat (1987), (in Russian).

[16] Bjorno L. Underwater acoustics and signal processing. London, D. Reidel Publishing (1981).

[17] Vainshtein L. A., Vakman D. E. Frequency division in the theory of oscillations and waves. Moscow, Nauka (1983), (in Russian).

[18] Cramer H., Leadbetter M. R. Stationary and related stochastic processes: sample function properties and their applications. New York, John Wiley \& Sons (1967).

[19] Bedrosian E. A product theorem for Hilbert transform. Proceedings of the IEEE. 51, 868-869 (1963).

[20] Javorskyi I., Yuzefovych R., Kurapov P. Periodically non-stationary analytic signals and their properties. 2018 IEEE 13th International Scientific and Technical Conference on Computer Sciences and Information Technologies (CSIT). 191-194 (2018). 


\title{
Кореляційні характеристики вузькосмугових періодично стаціонарних випадкових процесів
}

\author{
Яворський I. М. ${ }^{1,3}$, Курапов П. Р. ${ }^{1,2}$, Юзефович Р. М. ${ }^{1,2}$ \\ ${ }^{1}$ Фізико-механічний інститут ім. Г. В. Карпенка НАН Украӥни, \\ лабораторія вібродіагностики, \\ вул. Наукова, 5, Львів, 79060, Україна \\ ${ }^{2}$ Націоналъний університет "Львівсъка політехніка", \\ кафедра прикладної математики, \\ вул. С. Бандери, 12, Лввів, 79013, Україна \\ ${ }^{3}$ Технологічно-природничий університет, \\ Інститут телекомунікаиї та інформатики, \\ алея проф. С. Калісъкего, 7, Бидгощ, 85796, Польща
}

Розглянуто перетворення Гільберта вузькосмугових періодично корельованих сигналів. Отримано співідношення, що описують зв'язок між кореляційними компонентами сигналу та компонентами його перетворення Гільберта. На основі симульованих реалізацій проаналізовано залежність кореляційної функції перетворення Гільберта від декремента загасання кореляційного зв'язку модулюючих процесів.

Ключові слова: перетворення Гільберта, вузъкосмуговий періодично корельований випадковий сигнал, автокореляиійні та взаємокореляційні компоненти, коефічієнт загасання кореляційного зв'язку.

2000 MSC: $60 \mathrm{G} 35,94 \mathrm{~A} 12$

УдК: $621.391: 519.72$ 